To appear in The Astronomical Journal, March 2000

\title{
What Produced the Ultraluminous Supernova Remnant in NGC 6946?
}

\author{
Bryan C. Dunne, Robert A. Gruendl, and You-Hua Chu \\ Department of Astronomy, University of Illinois, 1002 West Green Street, Urbana, IL \\ 61801 \\ carolan@astro.uiuc.edu
}

\begin{abstract}
The ultraluminous supernova remnant (SNR) in NGC 6946 is the brightest known SNR in X-rays, 1000 times brighter than Cas A. To probe the nature of this remnant and its progenitor, we have obtained high-dispersion optical echelle spectra. The echelle spectra detect $\mathrm{H} \alpha$, [N II], and [O III] lines, and resolve these lines into a narrow (FWHM $\sim 20-40 \mathrm{~km} \mathrm{~s}^{-1}$ ) component from un-shocked material and a broad (FWHM $\sim 250 \mathrm{~km} \mathrm{~s}^{-1}$ ) component from shocked material. Both narrow and broad components have unusually high $[\mathrm{N} \mathrm{II}] / \mathrm{H} \alpha$ ratios, $\sim 1$. Using the echelle observation, archival HST images, and archival ROSAT X-ray observations, we conclude that the SNR was produced by a normal supernova, whose progenitor was a massive star, either a WN star or a luminous blue variable. The high luminosity of the remnant is caused by the supernova ejecta expanding into a dense, nitrogen-rich circumstellar nebula created by the progenitor.
\end{abstract}

Subject headings: (ISM:) supernova remnants — ISM: bubbles — stars: WolfRayet — ISM: H II regions — galaxies: individual (NGC 6946)

\section{Introduction}

NGC 6946 is a face-on Sc spiral galaxy at a distance of 5.1 Mpc (de Vaucouleurs 1979). Twenty-seven supernova remnant (SNR) candidates have been reported in this galaxy by Matonick \& Fesen (1997), using $\mathrm{H} \alpha$ and [S II] images. Because of the large distance to NGC 6946 and limited sensitivity of X-ray and radio detectors, it is difficult to confirm these SNR candidates. The most luminous SNR candidate in NGC 6946, listed as MF16 in 
Matonick \& Fesen (1997), is the only one of the 27 candidates confirmed with radio and X-ray observations (Van Dyk et al. 1994; Schlegel 1994).

MF16, at $\alpha=20^{\mathrm{h}} 35^{\mathrm{m}} 0.8, \delta=+60^{\circ} 11^{\prime} 30^{\prime \prime} .5$ (J2000), was the first known SNR in NGC 6946 because of its extremely high luminosities in both the X-ray and optical bands (Schlegel 1994; Blair \& Fesen 1994). It is the brightest known SNR in X-rays, 1000 times as luminous as the young Galactic remnant Cas $\mathrm{A}$ and $~ 3.5$ times as luminous as the young, ultraluminous SNR in NGC 4449 (Blair, Fesen, \& Schlegel 1997; Blair \& Fesen 1994). Its optical brightness is roughly the same as that of the NGC 4449 SNR and over 10 times greater than that of N49 (Blair \& Fesen 1994), the brightest SNR in the Large Magellanic Cloud (LMC). Such high luminosities are usually associated with young remnants, as is high velocity gas. Yet, gas with $\mathrm{V}_{\exp }>600 \mathrm{~km} \mathrm{~s}^{-1}$ has not been detected from MF16. Hubble Space Telescope (HST) images of this remnant show multiple loops. This has led Blair, Fesen, \& Schlegel (1999) to suggest that MF16 actually consists of colliding SNRs of different ages.

Intrigued by the extremely high luminosity and interesting morphology, we have obtained a high-dispersion echelle spectrum of MF16 to investigate its physical nature and the cause of its very high luminosity. The echelle spectrum clearly resolves the emission lines into a broad component and a narrow component, which presumably correspond to the shocked and un-shocked material, respectively. From the velocity profile of the $\mathrm{H} \alpha$ line and the $H S T$ F656N image, we are able to derive the mass and kinetic energy of the SNR. The echelle spectrum has also detected multiple nebular lines, allowing us to examine the diagnostic line ratios. We find that the $[\mathrm{N}$ II $] \lambda 6584$ to $\mathrm{H} \alpha$ line ratio is anomalously high for both the narrow and broad components. The $[\mathrm{N} \mathrm{II}] \lambda 6584 / \mathrm{H} \alpha$ ratio of the narrow line component is much higher than those of H II regions in NGC 6946 at similar galactrocentric distances. A high $[\mathrm{N} \mathrm{II}] / \mathrm{H} \alpha$ ratio is frequently seen in nebulae around Wolf-Rayet stars and luminous blue variables; for all such nebulae with good temperature measurements, an enhanced nitrogen abundance is found (e.g., Esteban et al. 1992; Smith et al. 1998). The high [N II] $] 6584 / \mathrm{H} \alpha$ ratio in the narrow component of the SNR MF16 is thus a significant discovery. To determine how unusual this high $[\mathrm{N} \mathrm{II}] \lambda 6584 / \mathrm{H} \alpha$ ratio is, we have compared MF16 to other SNR candidates in NGC 6946. We have also examined echelle spectra of eight SNRs in M33, and compared the $[\mathrm{NII}] \lambda 6584 / \mathrm{H} \alpha$ ratios for both the narrow and broad components. We have further explored the nature of MF16 by examining its X-ray spectra and comparing it to several SNRs in the LMC.

In this paper, we report on our observations and the results of our analysis of the ultraluminous SNR MF16 in NGC 6946. The details of the datasets are described in $\S 2$, our analysis is presented in $\S 3$, and the results are discussed in $\S 4$. 


\section{Observations and Archival Datasets}

The data we used to study the SNR MF16 in NGC6946 included available archived Hubble Space Telescope (HST) observations, high-dispersion echelle spectra, and archived ROSAT observations. For comparison, optical echelle spectra of a control group of eight SNRs in M33 and archived ROSAT observations of three SNRs in the LMC were also examined. All of the data were reduced using standard routines in $\mathrm{IRAF}^{1}$ and the STSDAS ${ }^{2}$ and $\mathrm{PROS}^{3}$ packages.

\subsection{HST WFPC2 Images}

HST Wide Field/Planetary Camera 2 (WFPC2) observations of MF16 (PI: Blair) took place on 1996 January 27 (UT). The remnant was centered on the PC chip for all exposures. A $2 \times 700$ s observation was made with the F656N filter $(\mathrm{H} \alpha)$ using $\mathrm{CR}$-split to improve cosmic ray rejection. A $2 \times 400$ s observation was made with the F439W filter (blue continuum) again using CR-split. A 700 s observation was made with the F555W filter (visual continuum); CRsplit was not used for this observation, making rigorous cosmic-ray removal difficult. The PC has a plate scale of $0^{\prime \prime} 0455$ pixel $^{-1}$ over a $36^{\prime \prime}$ field-of-view. The raw data were recalibrated using the most recent reference files recommended by the Space Telescope Science Institute. The flux calibration was obtained using the methods outlined on the WFPC2 website ${ }^{4}$. Images from the WFPC2 observations showing MF16 and neighboring regions are presented in Figure 1.

\subsection{Echelle Spectra}

Our high-dispersion spectra of MF16 were obtained using the echelle spectrograph on the 4-m telescope at Kitt Peak National Observatory (KPNO) on 1999 March 4 (UT). The $791 \mathrm{~mm}^{-1}$ echelle grating was used in combination with a $226 \mathrm{l} \mathrm{mm}^{-1}$ cross disperser and

\footnotetext{
${ }^{1}$ Image Analysis and Reduction Facility - IRAF is distributed by the National Optical Astronomy Observatories, which are operated by the Association of Universities for Research in Astronomy, Inc., under cooperative agreement with the National Science Foundation.

${ }^{2}$ Space Telescope Science Data Analysis System - http://ra.stsci.edu/

${ }^{3}$ PROS/XRAY Data Analysis System - http://hea-www.harvard.edu/PROS/pros.html

${ }^{4}$ http://www.stsci.edu/intrsuments/wfpc2/
} 
the long focus red camera to achieve a reciprocal dispersion of $3.5 \AA \mathrm{mm}^{-1}$ at $\mathrm{H} \alpha$. The spectra were imaged with the T2KB CCD detector. The pixel size is $24 \mu \mathrm{m}$, corresponding to $0^{\prime \prime} .24$ pixel $^{-1}$ along the slit and $\sim 3.7 \mathrm{~km} \mathrm{~s}^{-1}$ pixel $^{-1}$ along the dispersion axis. The SNR MF16 was observed for $600 \mathrm{~s}$ with a east-west oriented $15^{\prime \prime}$-long slit of width $2^{\prime \prime}$. The remnant was contained entirely within the slit. Despite the short exposure time, the sky lines are prominent and thus can be used to determine the instrumental FWHM, $17 \pm 1 \mathrm{~km} \mathrm{~s}^{-1}$, and to fine-tune the velocity calibration. The $\mathrm{H} \alpha-[\mathrm{N} \mathrm{II}] \lambda 6584$ region of the echellogram is presented in Figure 2.

The high-dispersion spectra of the SNRs in M33 were also obtained using the echelle spectrograph on the 4-m telescope at KPNO, but on 1986 September 16-17 (UT). Echellograms for M33-2, M33-4, M33-6, M33-8, M33-9, M33-11, M33-16, and M33-18 (notation from D'Odorico, Dopita, \& Benvenuti 1980) were obtained over the two nights. The $791 \mathrm{~mm}^{-1}$ echelle grating was used. A flat mirror, replacing the cross disperser, and a broad $\mathrm{H} \alpha$ post-slit filter were used to isolate a single order around the $\mathrm{H} \alpha$ line. These in combination with the UV camera achieved a reciprocal dispersion of $8.0 \AA \mathrm{mm}^{-1}$ at $\mathrm{H} \alpha$. The spectra were imaged with the TI-4 CCD detector. The pixel size was $15 \mu \mathrm{m}$, corresponding to 0 '.27 pixel $^{-1}$ along the slit and $\sim 5.5 \mathrm{~km} \mathrm{~s}^{-1}$ pixel $^{-1}$ along the dispersion axis. The M33 SNRs were observed with a east-west oriented long slit of width 3 .'3 for 1,800 s (M33-16), 2,400 s (M33-7), 2,700 s (M33-8, M33-9, M33-18), 3,000 s (M33-6), and 3,600 s (M33-2, M334, and M33-11). Th-Ar lamp comparison spectra were used to determine the instrumental FWHM, $31 \pm 1 \mathrm{~km} \mathrm{~s}^{-1}$. As an example of the echelle spectra obtained, the $\mathrm{H} \alpha-[\mathrm{N}$ II] region of M33-8's echellogram is presented in Figure 3.

\subsection{ROSAT PSPC Observations}

ROSAT observations of the galaxy NGC 6946 (PI: Schlegel) were made on 1992 June 11 (UT). The galaxy was observed for 36,713 s with the Position Sensitive Proportional Counter (PSPC). The sequence number of the observation is rp600272. A complete description of the observation can be found in Schlegel (1994). For comparison, we have also used ROSAT PSPC observations of LMC SNRs N49 (rp500062; 5,870 s; PI: Fink), N132D (rp500141; 6,212 s; PI: Hughes), and N157B (rp500131; 16,069 s; PI: Chu). The X-ray spectra are

presented in Figure 4. 


\section{Results and Analysis}

\subsection{Stellar and Interstellar Properties}

We have used the WFPC2 images to measure many of the basic properties of MF16. As measured using the F656N image (See Figure 1a), MF16 has an angular size of 0 ". $8 \times 1$ 1".2, corresponding to $20 \mathrm{pc} \times 30 \mathrm{pc}$ at a distance of $5.1 \mathrm{Mpc}$. In the F439W image (See Figure 1b), only a single continuum point source is projected within the remnant. To determine the origin of this point source, we first measured its apparent magnitude, $\mathrm{m}_{\mathrm{F} 439 \mathrm{~W}}=22.7 \pm 0.2 \mathrm{mag}$, using the "phot" package in IRAF. We then applied a correction of 0.66 mag given by Biretta et al. (1996) to convert to $\mathrm{m}_{\mathrm{B}}$. Using the $\mathrm{E}(\mathrm{B}-\mathrm{V})=0.52 \mathrm{mag}$ from Blair \& Fesen (1994) and a distance of $5.1 \mathrm{Mpc}$, we have determined that $\mathrm{M}_{\mathrm{B}}=-7.3 \mathrm{mag}$ for the point source ${ }^{5}$. The point source has a FWHM of $\sim 2$ pixels, and therefore it could be up to $\sim 2.5$ pc across. This size is too small for the point source to be an open cluster or OB association (Mihalas \& Binney 1981; Lucke \& Hodge 1970). The luminosity of the point source is consistent with a single class Ia supergiant or a multiple star system with fainter individual stars (Schmidt-Kaler 1982).

We have also explored the stellar and interstellar environments surrounding MF16. The remnant is in a rather unpopulated region of NGC 6946, many hundreds of parsecs north of a spiral arm. The F656N image shows that MF16 is not associated with any obvious H II region (See Figure 1a). A few faint point sources roughly $1^{\prime \prime}$ west of MF16 are visible in the WFPC2 F555W and F439W images (See Figure 1b). To determine whether these point sources constitute an $\mathrm{OB}$ association we have made photometric measurements. These measurements have high uncertainties because the sources are faint; the typical uncertainty is $0.5 \mathrm{mag}$ in $\mathrm{m}_{\mathrm{F} 439 \mathrm{~W}}$ and $0.2 \mathrm{mag}$ in $\mathrm{m}_{\mathrm{F} 555 \mathrm{~W}}$. If the extinction is negligible, the spectral types and luminosity classes of these stars range between B-early-A main-sequence stars and F-G class II bright giants (Schmidt-Kaler 1982). These spectral types are too late for this group of stars to be an OB association. On the other hand, due to the large uncertainities in the magnitude measurements, and hence colors, these stars could be brighter, have earlier spectral types, and may belong to an OB association. Nevertheless, the lack of an associated H II region indicates that either these stars have no ionizing power or there is no gas in the surrounding region; the extinction is therefore unknown. In either case, the association must be old, at least $\sim 10^{7}$ yr old.

The closest large structure to MF16 is an H II region centered $\sim 200$ pc to the northwest

\footnotetext{
${ }^{5}$ The F555W image is contaminaed by nebular [O III] emission from MF16, so the brightness of the point source cannot be accurately measured and visual magnitude cannot be derived.
} 
of the remnant at $\alpha=20^{\mathrm{h}} 34^{\mathrm{m}} 59.9, \delta=+60^{\circ} 11^{\prime} 36^{\prime \prime} \cdot 3$ (J2000). The location of the H II region is indicated on the F656N image (See Figure 1a). The F439W image also shows a stellar concentration in this same location (See Figure 1b). The morphology and spatial distribution of this H II region/stellar concentration are qualitatively similar to some H II regions/OB associations in the LMC (Lucke \& Hodge 1970). The stellar concentration is likely an OB association roughly $75 \mathrm{pc}$ across. This size is similar to the average OB association sizes for the LMC, 70 pc (Lucke \& Hodge 1970), and M31, 90 pc (Magnier et al. 1993). Blair, Fesen, \& Schlegel (1999) have raised the possibility that MF16 is associated with this stellar concentration. To include the stellar concentration and the stellar sources in and around MF16, the OB association would be $\sim 250$ pc across, far larger than the normal range indicated by LMC and M31 OB associations (Lucke \& Hodge 1970; Magnier et al. 1993). The OB association would also be required to extend over a region of space $\sim 120 \mathrm{pc}$ across that does not appear to contain a strong concentration of bright stars in the WFPC2 F439W image. This requirement is not compatible with the results of either older "by eye" OB association searches, such as Lucke \& Hodge (1970), or recent automated OB association searches using the Path Linkage Criterion or the "Friends of Friends" algorithm (Battinelli 1991; Wilson 1991). MF16 is therefore unlikely to be associated with the stellar concentration.

\subsection{Kinematic and Spectral Properties}

The echelle observation of the SNR MF16 detected the $\mathrm{H} \alpha$, [N II] $\lambda \lambda 6548,6584$, [O III $] \lambda \lambda 4959$, 5007 , and $[\mathrm{O} \mathrm{I}] \lambda 6300$ lines. The $[\mathrm{S} \mathrm{II}] \lambda \lambda 6716,6731$ lines are not detected as they were off the echelle blaze. The $\mathrm{H} \alpha$, [N II $] \lambda 6584$, and [O III $] \lambda 5007$ lines show a narrow component superposed on a broad component. The [N II] 66548 , [O III] $\lambda 4959$ and [O I] 66300 lines are too weak to detect the broad line component. We have determined the velocity widths of the broad and narrow components for the well-detected lines. The narrow components typically have FWHM of 20-40 $\mathrm{km} \mathrm{s}^{-1}$, while the broad components have FWHM $\sim 250 \mathrm{~km} \mathrm{~s}^{-1}$ (measured only for $\mathrm{H} \alpha$ and $[\mathrm{N} \mathrm{II}]$ ) and FWZI $\sim 425 \mathrm{~km} \mathrm{~s}^{-1}$ (See Table 1). We have taken the expansion velocity of the remnant to be the half-width at zero intensity of the $\mathrm{H} \alpha$ broad component and obtained $\mathrm{V}_{\exp } \approx 225 \mathrm{~km} \mathrm{~s}^{-1}$.

The majority of the $\mathrm{H} \alpha$, [N II] $\lambda 6584$, and [O III $] \lambda 5007$ emission from MF16 comes from

the broad component of the lines. In $\mathrm{H} \alpha$, the broad component is $\sim 1.5$ times as strong as the narrow component. In $[\mathrm{N}$ II $] \lambda 6584$, the broad component is $\sim 1.8$ times as strong as the narrow component. In $[\mathrm{O}$ III $] \lambda 5007$, the broad component is only slightly stronger than the narrow component. The most striking and unusual feature of the spectra is that the 
total $[\mathrm{N} \mathrm{II}] \lambda 6584$ flux is comparable to the $\mathrm{H} \alpha$ flux. The $[\mathrm{N} \mathrm{II}] \lambda 6584 / \mathrm{H} \alpha$ ratio of the broad component is near unity while that of the narrow component is slightly less. A full detailing of comparative flux levels can be found in Table 2 .

\subsection{Physical Properties}

We have measured a total flux for MF16 of $~ 5,000$ counts from the 1,400 s F656N WFPC2 image using the "polyphot" routine in IRAF. From the method for narrow-band photometry outlined on the WFPC2 website $^{6}$, we have determined the $\mathrm{H} \alpha$ flux to be $1.5 \times 10^{-14} \mathrm{erg} \mathrm{s}^{-1} \mathrm{~cm}^{-2}$. Using the $\mathrm{E}(\mathrm{B}-\mathrm{V})$ value of $0.52 \mathrm{mag}$ and the distance value of $5.1 \mathrm{Mpc}$, we find a total $\mathrm{H} \alpha$ luminosity of $1.4 \times 10^{38} \mathrm{erg} \mathrm{s}^{-1}$. This value is consistent with the luminosity reported by Blair \& Fesen (1994), $1.9 \times 10^{38} \mathrm{erg} \mathrm{s}^{-1}$, within their error limit of $25 \%$. Under the assumption that the broad line component originates from the expanding SNR shell, the ratio between the broad and narrow components suggests that $\sim 8.5 \times 10^{37} \mathrm{erg} \mathrm{s}^{-1}$ of the total $\mathrm{H} \alpha$ luminosity originates from the expanding SNR shell. We have used this broad component $\mathrm{H} \alpha$ luminosity to determine the mass and kinetic energy of the SNR shell. We have assumed an ellipsoidal shell structure with a third axis of $25 \mathrm{pc}$ and a shell thickness of $1 \%$ of the radius. We have also used the simple assumption that the remnant is composed of singly ionized hydrogen and helium. Using the electron density determined by Blair, Fesen, \& Schlegel (1999), $410 \pm 100 \mathrm{~cm}^{-3}$, we find a filling factor of $0.21_{-.08}^{+.15}$, a mass of $640_{-130}^{+200} \mathrm{M}_{\odot}$ and a kinetic energy of $3.2_{-0.7}^{+1.0} \times 10^{50} \mathrm{erg}$ for the SNR shell (See Table 3).

\subsection{X-Ray Properties}

We have used the ROSAT PSPC observation of NGC 6946 to extract the X-ray spectrum of MF16. To fit the X-ray spectrum, we applied both a thin plasma emission model (Raymond \& Smith 1977) and a power-law model. We found a power-law model with energy index $\alpha=3.7 \pm 0.6$ and absorption column density $\mathrm{N}_{\mathrm{H}}=5.6_{-1.1}^{+1.5} \times 10^{21} \mathrm{~cm}^{-2}$ to be the best fit for the ROSAT data (See Figure 4a) with $\min \left(\chi^{2}\right)=25.5$, half that of the best thin plasma emission model fit. Our power-law fit is consistent with that of Schlegel (1994), who concluded that the X-ray spectrum of MF16 could be fitted equally well by several simple models, not including the Raymond-Smith thin plasma emission model. The ambiguity in the model fit was attributed to the low spectral resolution and the very soft energy band of

${ }^{6}$ http://www.stsci.edu/instruments/wfpc2/Wfpc2_faq/wfpc2_nrw_phot_faq.html 
the PSPC. We note that the PSPC spectrum of MF16 has a high-energy tail above $2 \mathrm{keV}$, as shown in Figure 4. This high-energy emission has been confirmed by an ASCA observation, which shows a complex spectrum that requires multiple emission components (Schlegel \& Blair 1998).

\subsection{M33 Remnants}

We have examined the echelle spectra of the M33 SNRs by the same methods used for the echelle spectra of MF16. The broad line components of the M33 remnants show $[\mathrm{N} \mathrm{II}] \lambda 6584 / \mathrm{H} \alpha$ ratios of $0.3-0.55$. The narrow line components show $[\mathrm{N} \mathrm{II}] \lambda 6584 / \mathrm{H} \alpha$ ratios of 0.15-0.35, similar to the H II regions near the SNRs. A complete summary of the M33 SNRs can be found in Table 4 . These $[\mathrm{N}$ II $] \lambda 6584 / \mathrm{H} \alpha$ ratios are significantly lower than the $[\mathrm{N}$ II] $] 6584 / \mathrm{H} \alpha$ ratios of the remnant MF16.

Even the remnants with velocity widths similar to those of the SNR MF16 have weaker $[\mathrm{N} \mathrm{II}] \lambda 6584 / \mathrm{H} \alpha$ ratios than those in MF16. M33-6, M33-8, M33-9 have H $\alpha$ broad line component FWHM $\sim 270 \mathrm{~km} \mathrm{~s}^{-1}$ and $\mathrm{H} \alpha$ narrow line component FWHM $\sim 40 \mathrm{~km} \mathrm{~s}^{-1}$, very close to the $\mathrm{H} \alpha$ velocity widths of MF16. The broad line component [N II] $\lambda 6584 / \mathrm{H} \alpha$ ratios for the M33-6, M33-8, and M33-9 are, however, only half as strong as those in MF16, and the narrow line component $[\mathrm{NII}] \lambda 6584 / \mathrm{H} \alpha$ ratios are only one-third as strong as those in the ultraluminous SNR MF16.

\section{Discussion}

\subsection{Luminosity Consideration}

The SNR MF16 has an extraordinary luminosity; it is comparable in optical brightness to the young, ultraluminous SNR in NGC 4449 and $~ 3.5$ times brighter in X-rays. Despite this, the supernova does not appear to have been highly energetic. The kinetic energy

of the remnant shell, $\sim 3 \times 10^{50} \mathrm{erg}$, is similar to the shell kinetic energies of $\sim 10^{50} \mathrm{erg}$ determined by Williams et al. (1997, 1999a) for remnants in the Magellanic Clouds, ruling out the necessity of a hypernova remnant (Wang 1999) or other exotic object with explosion energy $\gtrsim 10^{53} \mathrm{erg}$. The remnant does not appear very young $\left(\sim 10^{2} \mathrm{yr}\right)$ because it has a low expansion velocity and a moderate size. If we assume that this SNR is formed by a single supernova in a homogeneous medium and that the SNR is in an adiabatic expansion stage, we may use the Sedov (1959) solution to derive an age of $\sim 25,000 \mathrm{yr}$. This age is an order of magnitude greater than the upper age limit determined by Blair \& Fesen $(1994), \leq 3,500$ 
yr. While we make no claim the Sedov solution accurately describes MF16, it demonstrates that the remnant could be millennia in age.

If MF16 is not highly energetic or very young, then we must find another explanation to generate the luminosity of the remnant. A possible mechanism for the luminosity could be the interaction of the expanding SNR shell with dense material around the SNR. The young, ultraluminous SNR in NGC 4449, which has optical luminosity comparable to the SNR MF16 (Blair \& Fesen 1994), is luminous because it is expanding into a nearby H II region (Blair, Kirshner, \& Winkler 1983). MF16 may also be luminous because it is expanding into dense nearby material. As emissivity $\propto \mathrm{N}_{e}^{2} \mathrm{~V}$, where $\mathrm{N}_{e}$ is the electron density and $\mathrm{V}$ is the emitting volume, a moderate increase in the density can greatly increase the luminosity. We note that the existence of a bright narrow line component in MF16 indicates the existence of such dense circumstellar material.

The interaction of MF16's SNR shell with dense circumstellar material can explain the optical luminosity, but it is not clear whether or not this interaction can produce the high X-ray luminosity. To further explore the X-ray properties of MF16, we have compared the ROSAT PSPC observations of this remnant to those of the LMC SNRs N49, N132D, and N157B from Williams et al. (1999b). In Figure 4, we compare MF16 to the LMC SNRs. These three LMC SNRs have been previously observed by the Einstein Solid State Spectrometer. From these Einstein data, Clark et al. (1982) find that the X-ray spectra of N49 and N132D are best described by a thin plasma emission model and that the spectrum of N157B is best described by a power-law model. In $\S 3.4$, we determined that MF16's $\mathrm{X}$-ray spectrum is best fitted by a power-law model and shows significant emission above $2 \mathrm{keV}$. The spectral characteristics are most similar to those of N157B. Based on its radio and X-ray spectra, N157B has been identified as a Crab-type SNR (Mills, Turtle, \& Watkinson 1978; Clark et al. 1982; Mathewson et al. 1983), and it is known to contain a pulsar (Marshall et al. 1998). Based on the spectral similarities between MF16 and N157B, we suspect that a significant portion of the X-ray luminosity of MF16 is contaminated by a non-thermal-plasma source. To determine the nature of the non-thermal-plasma source, we need high spatial resolution and high spectral resolution X-ray observations combined with a sensitive timing experiment. At present, it is premature to determine the real fraction of thermal plasma emission in the X-ray spectrum of MF16.

\subsection{Significance of the $[\mathrm{N}$ II $] \lambda 6584 / \mathrm{H} \alpha$ Ratio}

Much can be learned from the $[\mathrm{N}$ II $] \lambda 6584 / \mathrm{H} \alpha$ ratios of the broad and narrow line components of an SNR's optical spectrum by comparing them to those of nearby H II regions. 
We use the SNRs of M33 to illustrate this effect. As described in $\S 3.5$, most of the observed SNRs in M33 are adjacent to $\mathrm{HII}$ regions, and the observed $[\mathrm{N} \mathrm{II}] \lambda 6584 / \mathrm{H} \alpha$ ratios in the narrow components at the SNRs are similar to those in the adjacent HII regions. This indicates that the narrow components originate in the un-shocked material surrounding the SNRs. The broad components of the SNRs are associated with the expanding shells of shocked gas. As the shocked gas has higher temperatures, and as forbidden line strengths increase steeply with temperature, the forbidden/recombination line ratios should be higher in shocked gas with higher temperatures. Indeed, the M33 SNRs show higher $[\mathrm{N}$ II $] \lambda 6584 / \mathrm{H} \alpha$ ratios in the broad components than in the narrow components.

The SNR MF16 has unusually high $[\mathrm{N}$ II $] \lambda 6584 / \mathrm{H} \alpha$ ratios. The total $[\mathrm{N}$ II $] \lambda 6584 / \mathrm{H} \alpha$ ratio of MF16 is roughly twice the average $[\mathrm{N} \mathrm{II}] \lambda 6584 / \mathrm{H} \alpha$ ratio for other SNR candidates in NGC 6946, 0.45 (Matonick \& Fesen 1997). Both its broad line component and the narrow line component have higher $[\mathrm{N}$ II $] \lambda 6584 / \mathrm{H} \alpha$ ratios than the observed M33 SNRs. We do not have echellograms of any nearby $\mathrm{H}$ II regions for a direct comparison; however, several H II regions at galactocentric distances of $2.5 \mathrm{kpc}$ and $4.5 \mathrm{kpc}$, similar to that of MF16 (3.7 kpc), have been observed by McCall, Rybski, \& Shields (1985) and can be compared with the narrow line component of the remnant. These H in regions in NGC 6946 have [N II] $6584 / \mathrm{H} \alpha$ ratios $\sim 0.3$, similar to those seen in H II regions and SNR narrow line components in M33, but are much lower than that of the narrow line component of the NGC6946 remnant, 0.8.

High $[\mathrm{N} \mathrm{II}] / \mathrm{H} \alpha$ ratios can be associated with shock excitation or an enhanced nitrogen abundance. While the shock excitation may be partially responsible for the high $[\mathrm{N}$ II $] \lambda 6584 / \mathrm{H} \alpha$ ratios in the broad line component, shock excitation cannot be responsible for the narrow line component. The FWHM of the narrow component of the $[\mathrm{N} \mathrm{II}] \lambda 6584$ line is only $25 \mathrm{~km} \mathrm{~s}^{-1}$ for the SNR MF16, implying a gas motion of $\sim 13 \mathrm{~km} \mathrm{~s}^{-1}$, which is only slightly larger than the isothermal sound velocity of a $10^{4} \mathrm{~K}$ gas. We are left with the inevitable alternative that an enhanced nitrogen abundance causes the high $[\mathrm{N}$ II $] \lambda 6584 / \mathrm{H} \alpha$ ratio in the narrow line component of the remnant.

Elevated nitrogen abundance and high $[\mathrm{N}$ II $] / \mathrm{H} \alpha$ ratios are a hallmark of stellar ejecta nebulae associated with nitrogen-enhanced Wolf-Rayet (WN) stars and luminous blue variables (LBVs) (e.g., Esteban et al. 1992; Smith et al. 1998). The SNR MF16 may be interacting with a circumstellar nebula produced by the supernova's progenitor. The expansion velocity of this nebula, $\sim 13 \mathrm{~km} \mathrm{~s}^{-1}$, implied by the FWHM of the narrow [N II] $\lambda 6584$ component, is near the low end of the range of expansion velocities observed in ring nebulae around massive stars (Chu, Weis, \& Garnett 1999).

SNRs interacting with the progenitors' ejecta nebulae can be observed only when the SNRs are young, as old SNRs' evolution will be dominated by the distribution of ambient 
interstellar medium. This interaction is actually commonly seen in young SNRs. The two youngest known SNRs in the LMC, SN1987A and SNR 0540-69.3, both show a circumstellar nebula with an enhanced [N II]/H $\alpha$ ratio (Burrows et al. 1995; Caraveo, Mignani, \& Bignami 1998). The youngest SNR in our Galaxy, Cas A, also shows traces of its progenitor's ejecta. Cas A contains bright, slow-moving knots of gas called quasi-stationary flocculi (QSF). The QSFs have $[\mathrm{NII}] / \mathrm{H} \alpha$ ratios of $1-4$, and nitrogen abundances enhanced by a factor of 10 (Kirshner \& Chevalier 1977). The QSFs have been suggested to be ejected by a WN progenitor of the supernova (Peimbert \& van den Bergh 1971; Chevalier \& Kirshner 1978). We propose that MF16 is a young SNR, $\lesssim 10^{3} \mathrm{yr}$, created by the supernova explosion of a WN or LBV star, and that it is interacting with the ring nebula produced by the progenitor.

\subsection{Colliding SNRs vs. Single SNR}

It has been suggested that MF16 is the product of colliding SNRs of different ages (Blair, Fesen, \& Schlegel 1999). This colliding SNR hypothesis is based on the multipleloop morphology seen in the F656N WFPC2 image (See Figure 5). The colliding SNR hypothesis requires the detonation of at least two massive stars in close proximity to one another in both space and time. Blair, Fesen, \& Schlegel (1999) support this possibility by suggesting that MF16 is embedded in an OB association. As discussed in $\S 3.1$, any association in this region would be $\gtrsim 10^{7}$ yr old. We would therefore expect the O-early-B type stars of this association to have "burned out" already. This is also the disintegration timescale of OB associations (Scheffler \& Elsässer 1988). Thus, any association in the region is comprised of mid- to late-B type stars and is mixing into the general stellar population. We suggest that the probability of multiple supernovae occurring in this environment, with the proper spatial and temporal proximity to one another, is very low.

The multiple-loop morphology of MF16 is also consistent with a single SNR hypothesis. During the evolution of a massive star, the main-sequence winds, stellar ejecta, supergiant winds, and/or Wolf-Rayet winds shape multiple shells of ambient material around the evolving star (Marston 1995; García-Segura et al. 1996a,b). This effect is clearly seen in the nebula NGC6888, which contains a WN star surrounded by a nitrogen-enriched circumstellar bubble of of size $12 \times 6 \mathrm{pc}$ and an interstellar bubble of diameter 28 pc (Cappa et al. 1996). As a massive star inside such a nebula explodes as a supernova, the resultant SNR

will have a multiple-loop morphology. We therefore suggest that a single SNR hypothesis is favored over a colliding SNR hypothesis for MF16. 


\section{Conclusions}

We conclude that the ultraluminous SNR MF16 in NGC6946 is a normal SNR expanding into a complex ring nebula formed by a massive progenitor, specifically a WN star or LBV. Such a nebula is expected to contain a fragmented circumstellar bubble of stellar ejecta surrounded by a larger interstellar bubble of ambient material swept up by stellar winds (García-Segura et al. 1996a,b). The optical luminosity of the remnant is caused by the interaction of the SNR shell with these dense shells of material in the nebula. The multiple-loop morphology seen in the F656N WFPC2 image is a result of this interaction. Since the supernova exploded into the cavity of this nebula, the SNR shell would have expanded rapidly through the fragmented circumstellar bubble until it encountered the outer interstellar bubble. The remnant is therefore still young, $\lesssim 10^{3} \mathrm{yr}$, and the interior of the SNR contains dense clumps of stellar ejecta being ablated by the SNR shock and evaporated by the hot medium of the SNR interior. We further suggest that because the progenitor was either a WN star or an LBV, its circumstellar material is nitrogen-rich, leading to the strong $[\mathrm{N} \mathrm{II}] / \mathrm{H} \alpha$ ratio. Similar high luminosity is expected when the ejecta of SN1987A hits the circumstellar rings, which have strong $[\mathrm{N} \mathrm{II}] / \mathrm{H} \alpha$ ratios (Burrows et al. 1995). Future observations with a spectroscopic instrument able to resolve the nebula, such as the Space Telescope Imaging Spectrograph, would allow the spectral properties of individual sections of the SNR MF16 to be probed, resolving many of the outstanding questions of this most interesting supernova remnant.

We would like to thank William Blair for his useful communications in preparing this paper and for being a co-observer on the M33 echelle data. We also thank Schuyler Van Dyk for being a co-observer on the MF16 echelle data, Rosie Chen for valuable discussions in analyzing the WFPC2 data, and Rosa Williams for access to her X-ray atlas of LMC SNR. Finally, we thank the anonymous referee for his/her helpful comments. This research is supported by NASA grant NAG 5-7003. 


\section{REFERENCES}

Battinelli, P. 1991, A\&A, 244, 69

Biretta, J. A., et al. 1996, WFPC2 Instrument Handbook, Version 4.0 (Baltimore: STScI). (c) 1996 by STScI

Blair, W.P., Fesen, R.A., \& Schlegel, E.M. 1997, AAS, 190, 2706

Blair, W.P., Fesen, R.A., \& Schlegel, E.M. 1999, submitted to AJ

Blair, W.P., \& Fesen, R.A. 1994, ApJ, 424, L103

Blair, W.P., Kirshner, R.P., \& Winkler, P.F. 1983, ApJ, 272, 84

Burrows, C.J., et al. 1995, ApJ, 452, 680

Cappa, C.E., Dubner, G.M., Rogers, C., \& St-Louis, N. 1996, AJ, 112, 1104

Caraveo, P.A., Mignani, R., \& Bignami, G.F. 1998, MmSAI, 69, 1061

Chevalier, R.A., \& Kirshner, R.P. 1978, ApJ, 219, 931

Chu, Y.-H., Weis, K., \& Garnett, D.R. 1999, AJ, 117, 1433

Clark, D.H. et al. 1982, ApJ, 255, 440

D’Odorico, S., Dopita, M.A., \& Benvenuti, P. 1980, A\&AS, 40, 67

de Vaucouleurs, G. 1979, ApJ, 227, 729

Esteban, C., Vilchez, J.M., Smith, L.J., \& Clegg, R.E.S. 1992, A\&A, 259, 629

García-Segura, G., Mac Low, \& M.-M., Langer, N. 1996a, A\&A, 305, 229

García-Segura, G., Langer, N., \& Mac Low, M.-M. 1996b, A\&A, 316, 133

Kirshner, R.P., \& Chevalier, R.A. 1977, ApJ, 218, 142

Lucke, P.B., \& Hodge, P.W., 1970, AJ, 75, 171

Magnier, E.A., et al. 1993, A\&A, 278, 36

Marshall, F.E., Gotthelf, E.V., Zhang, W., Middleditch, J., \& Wang, Q.D. 1998, ApJ, 499, L179

Marston, A.P. 1995, AJ, 109, 1839 
Mathewson, D.S. et al. 1983, ApJS, 51, 345

Matonick, D.M., \& Fesen, R.A. 1997, ApJS, 112, 49

McCall, M.L., Rybski, P.M., \& Shields, G.A. 1985, ApJS, 57, 1

Mihalas, D.M., \& Binney, J. 1981, Galactic Astronomy, 2nd Edition, (San Francisco: Freeman)

Mills, B.Y., Turtle, A.J., \& Watkinson, A. 1978, MNRAS, 185, 263

Peimbert, M., \& van den Bergh, S., 1971, ApJ, 167, 223

Raymond, J.C., \& Smith, B.W. 1977, ApJS, 35, 419

Sedov, L.I. 1959, Similarity and Dimensional Methods in Mechanics, (New York: Academic)

Schlegel, E.M. 1994, ApJ, 424, L99

Schlegel, E.M., \& Blair, W.P. 1998, AAS, 193.0819

Schmidt-Kaler, Th. 1982, in Landolt-Bornstein: Numerical Data and Functional Relationships in Science and Technology, vol 2b, eds Schaifers, K., Voigt, H.H. (Berlin: Springer)

Scheffler H., Elsässer, H. 1988, Physics of the Galaxy and Interstellar Matter, (Berlin: Springer-Verlag)

Smith, L.J., Nota, A., Pasquall, A., Leitherer, C., Clampin, M., \& Crother, P.A. 1998, ApJ, 503,278

Van Dyk, S.D., Sramek, R.A., Weiler, K.W., Hyman, S.D., \& Virden, R.E. 1994, ApJ, 425, L77

Wang, Q.D. 1999, ApJ, 517, L27

Williams, R.M., et al. 1997, ApJ, 480, 618

Williams, R.M., et al. 1999a, ApJ, 514, 798

Williams, R.M., et al. 1999b, in preparation

Wilson, C.D. 1991, AJ, 101, 1663 

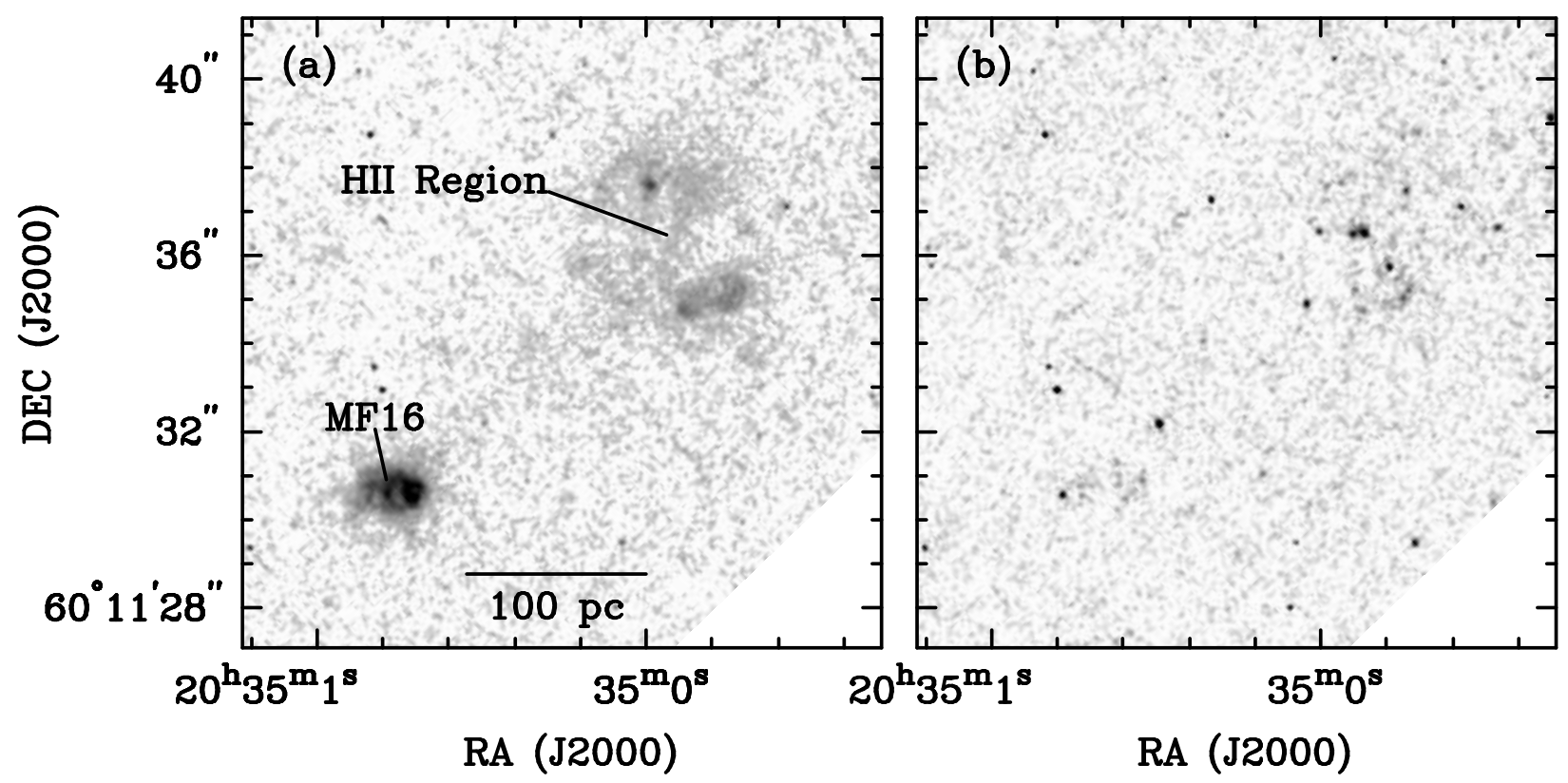

RA (J2000)

Fig. 1.- WFPC2 images of MF16 and surrounding region: (a) F656N image (H $\alpha$ ), (b) F439W image (blue continuum). The location of MF16 and a nearby H II region are indicated in (a). 


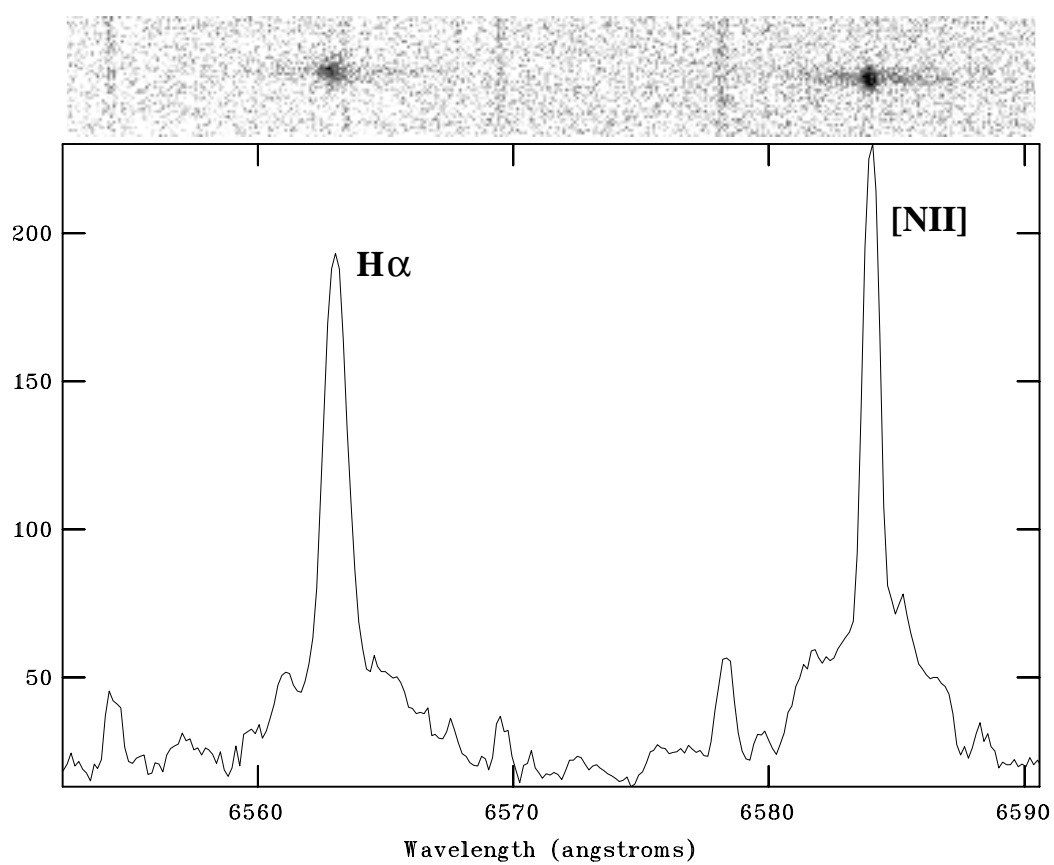

Fig. 2.- Echellogram and corresponding spectrum of MF16 with $\mathrm{H} \alpha$ and [N II] lines shown. Both a narrow and broad line component of the emission from the SNR are evident.

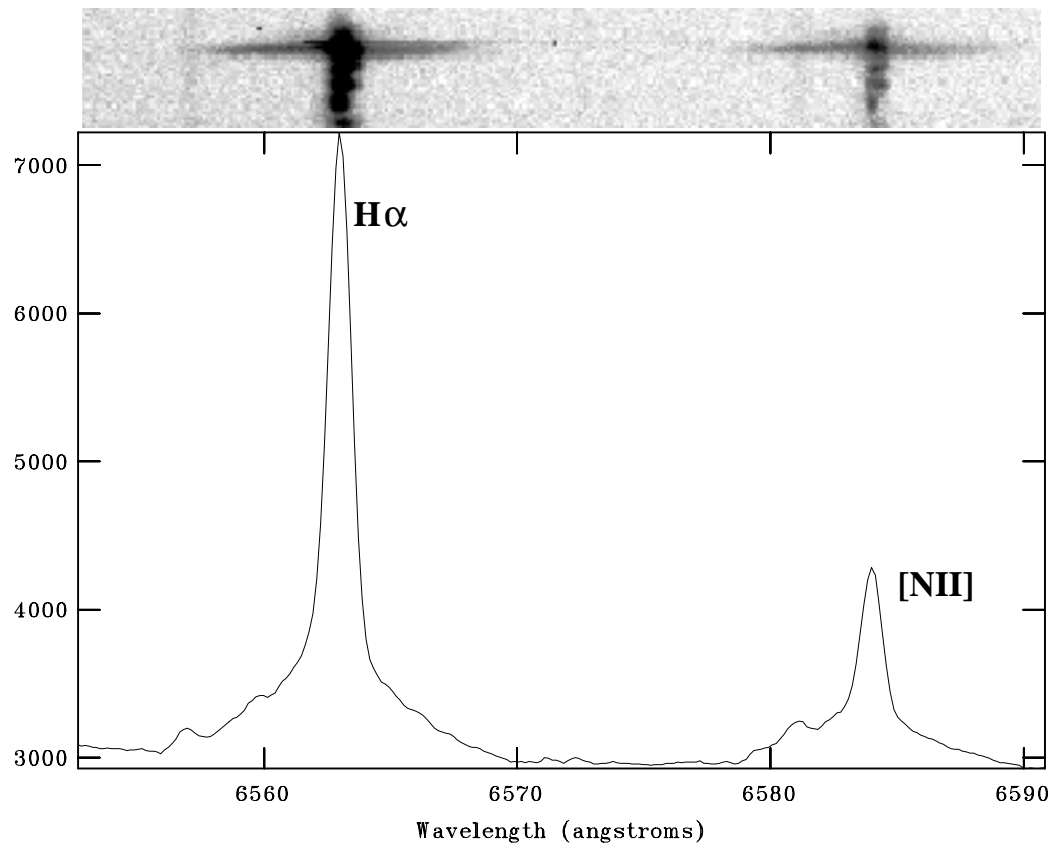

Fig. 3.- Echellogram and corresponding spectrum of M33-8 with $\mathrm{H} \alpha$ and [N II] lines shown. As in Figure 2, both a narrow and broad line component of the emission from the SNR are evident. 

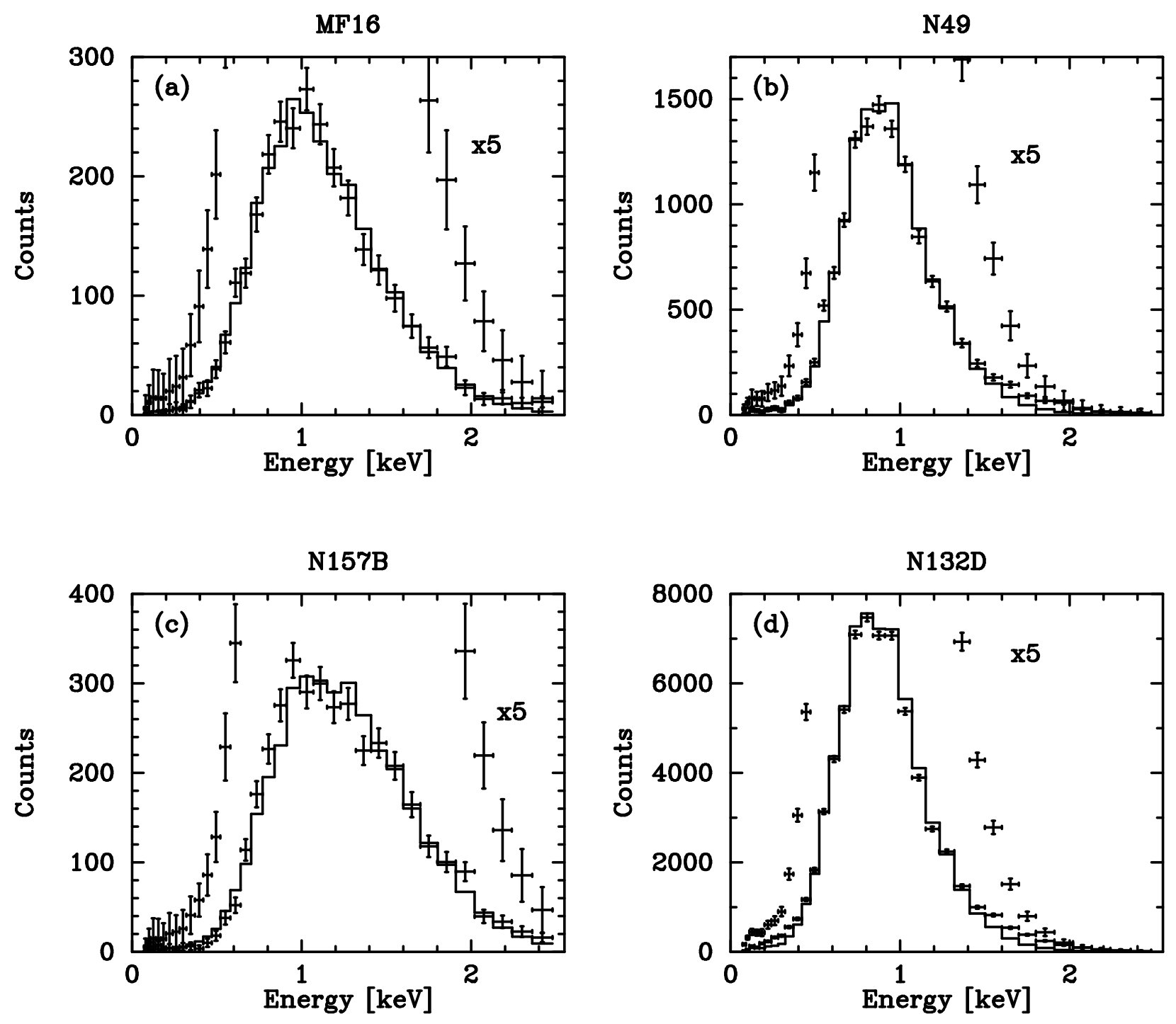

Fig. 4.- ROSAT PSPC X-Ray spectra for (a) MF16, (b) N49, (c) N157B, and (d) N132D. In each panel, the spectrum is plotted twice, with the upper plot exaggerated by a factor of 5 to emphasize the high-energy tail above $2 \mathrm{keV}$. The lower plot shows the best-fit model: power-law model for MF16 and N157B, Raymond \& Smith's (1977) thin plasma emission model for N49 and N132D. 


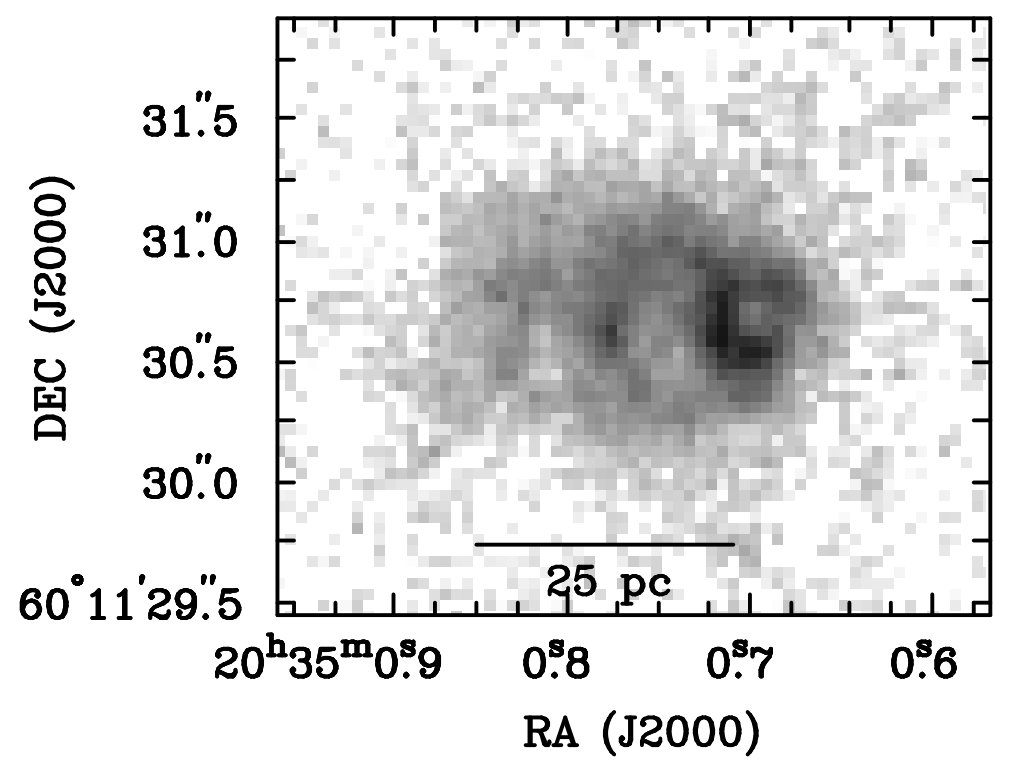

Fig. 5.- Narrow field-of-view WFPC2 F656N (H $\alpha$ ) image of MF16 to show multiple-loop morphology. 
Table 1: MF16 Spectral Velocity Widths

\begin{tabular}{lcc}
\hline \hline & $\begin{array}{c}\text { Broad } \\
\text { Component }\end{array}$ & $\begin{array}{c}\text { Narrow } \\
\text { Component }\end{array}$ \\
\hline $\mathrm{H} \alpha$ FWHM $\left(\mathrm{km} \mathrm{s}^{-1}\right)$ & 285 & 42 \\
{$[\mathrm{~N} \mathrm{II}] \lambda 6584 \mathrm{FWHM}\left(\mathrm{km} \mathrm{s}^{-1}\right)$} & 250 & 25 \\
{$[\mathrm{O}$ III $] \lambda 5007 \mathrm{FWHM}\left(\mathrm{km} \mathrm{s}^{-1}\right)$} & - & 22 \\
$\mathrm{H} \alpha$ FWZI $\left(\mathrm{km} \mathrm{s}^{-1}\right)$ & 450 & - \\
{$[\mathrm{N} \mathrm{II}] \lambda 6584 \mathrm{FWZI}\left(\mathrm{km} \mathrm{s}^{-1}\right)$} & 400 & - \\
{$[\mathrm{O}$ III $] \lambda 5007 \mathrm{FWZI}\left(\mathrm{km} \mathrm{s}^{-1}\right)$} & 430 & - \\
\hline
\end{tabular}

Table 2: MF16 Comparative Flux Levels

\begin{tabular}{lc}
\hline \hline & Ratio \\
\hline$[\mathrm{N}$ II $] \lambda 6584 / \mathrm{H} \alpha$ broad & 1.0 \\
{$[\mathrm{~N}$ II $] \lambda 6584 / \mathrm{H} \alpha$ narrow } & 0.8 \\
$\mathrm{H} \alpha$ broad/narrow & 1.5 \\
{$[\mathrm{~N}$ II $] \lambda 6584 \mathrm{broad} /$ narrow } & 1.8 \\
{$[\mathrm{O}$ III $] \lambda 5007 \mathrm{broad} /$ narrow } & $\sim 1$ \\
\hline
\end{tabular}

Table 3: Physical Parameters Of MF16's SNR Shell

\begin{tabular}{lc}
\hline \hline Parameter & Value \\
\hline Luminosity $(\mathrm{H} \alpha)$ & $1.4 \times 10^{38} \mathrm{erg} \mathrm{s}^{-1}$ \\
Electron Density & $410 \mathrm{~cm}^{-3}$ \\
Filling Factor & 0.21 \\
Mass & $640 \mathrm{M}_{\odot}$ \\
Kinetic Energy & $3.2 \times 10^{50} \mathrm{erg}$ \\
\hline
\end{tabular}

${ }^{a}$ From Blair, Fesen, \& Schlegel (1999) 
Table 4: M33 SNRs Flux Ratios \& Spectral Velocity Widths

\begin{tabular}{|c|c|c|c|c|c|c|c|}
\hline & \multicolumn{3}{|c|}{$\begin{array}{c}\text { Broad } \\
\text { Component }\end{array}$} & \multicolumn{3}{|c|}{$\begin{array}{c}\text { Narrow } \\
\text { Component }\end{array}$} & \multirow{3}{*}{$\begin{array}{c}\text { H II } \\
\text { Region } \\
{[\mathrm{N} \mathrm{II}]^{\prime /} \mathrm{H} \alpha}\end{array}$} \\
\hline & \multirow[t]{2}{*}[\mathrm{N}\mathrm{III}]{$^{q /} \mathrm{H} \alpha$} & \multicolumn{2}{|c|}{ FWHM } & \multirow[t]{2}{*}[\mathrm{N}\mathrm{III}]{$^{a / H} \alpha$} & \multicolumn{2}{|c|}{ FWHM } & \\
\hline & & $\begin{array}{c}\mathrm{H} \alpha \\
\left(\mathrm{km} \mathrm{s}^{-1}\right)\end{array}$ & $\begin{array}{c}{[\mathrm{N} \mathrm{II}]^{\mathrm{a}}} \\
\left(\mathrm{km} \mathrm{s}^{-1}\right)\end{array}$ & & $\begin{array}{l}\mathrm{H} \alpha \\
\mathrm{km} \mathrm{s}^{-}\end{array}$ & $\begin{array}{c}{[\mathrm{N} \mathrm{II}]^{\mathrm{a}}} \\
\left(\mathrm{km} \mathrm{s}^{-1}\right)\end{array}$ & \\
\hline M33-2 & 0.3 & 190 & 190 & 0.15 & 45 & 30 & 0.15 \\
\hline M33-4 & 0.3 & 140 & 145 & 0.2 & 40 & 30 & 0.3 \\
\hline M33-6 & 0.5 & 255 & 240 & 0.25 & 40 & 25 & 0.25 \\
\hline M33-8 & 0.5 & 275 & 265 & 0.3 & 40 & 40 & 0.3 \\
\hline M33-9 & 0.45 & 270 & 255 & 0.25 & 40 & 40 & 0.2 \\
\hline M33-11 & 0.55 & 140 & 150 & 0.35 & 50 & 50 & 0.25 \\
\hline M33-16 & - & - & - & 0.2 & 60 & 50 & 0.2 \\
\hline M33-18 & 0.4 & 145 & 140 & 0.25 & 30 & 25 & 0.15 \\
\hline
\end{tabular}

${ }^{a}[\mathrm{~N}$ II $] \lambda 6584$ 